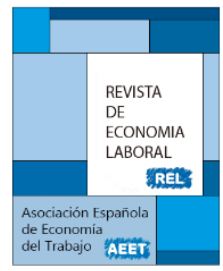

\title{
EL TRABAJO INFORMAL EN CHILE, ¿OPCIÓN VOLUNTARIA O CONDICIÓN OBLIGADA?
}

\author{
Víctor Andrés León Ossandón \\ Ingeniero Civil Industrial, \\ Doctor en Economía y Gestión Empresarial
}

Recibido Julio 2018; Aceptado Diciembre 2018

\section{Resumen}

El presente artículo estudia la informalidad laboral en Chile, bajo la hipótesis de que este tipo de actividades son heterogéneas, en el sentido que albergan actividades precarias, como opción obligada, y también a quienes están optando por un trabajo informal debido a consideraciones de beneficio - costo.

Se diseña una fórmula de medición de informalidad laboral y se utiliza un método de variables instrumentales, aplicadas a un modelo de selección y tratamiento con presencia de heterogeneidad esencial, donde se asimila el concepto de tratamiento al de elección de un trabajo formal.

Como resultado, se obtienen indicios que las personas están respondiendo al tratamiento, basados en ganancias o recompensas idiosincráticas y se encuentran revelando una variación conductual que las lleva a elegir la modalidad de trabajo. Sin embargo, cuando se prueba en personas del primer quintil socioeconómico, se obtiene un resultado menos concluyente y existiría una evidencia más débil sobre la presencia de barreras de acceso a trabajos formales.

Palabras clave: Chile, trabajo informal, efectos de tratamiento, heterogeneidad esencial. Clasificación JEL: C31, E26, O17

\begin{abstract}
This article studies labor informality in Chile, under the hypothesis that this type of activities is heterogeneous, in the sense that they have precarious activities, as an obligatory option, and to those who are opting for an informal work due to benefit-cost considerations.

A formula for measuring labor informality is designed and a method of instrumental variables is used, applied to a selection and treatment model with the presence of essential heterogeneity, where the concept of treatment is assimilated to that of choosing a formal job.

As a result, indications are obtained that people are responding to treatment, based on idiosyncratic gains or rewards, and are revealing a behavioral variation that leads them to choose the mode of work. However, when tested on people in the first socioeconomic quintile, a less conclusive result is obtained and there would be weaker evidence about the presence of barriers to access to formal jobs.
\end{abstract}

Key words: Chile, informal work, treatment effect, essential heterogeneity.

JEL Classification : C31, E26, O17

C Revista de Economía Laboral 


\section{Introducción}

La informalidad laboral está cobrando relevancia en Chile, pues si bien, registra una de las tasas más bajas del continente, se encuentra experimentando una creciente visibilidad que ha motivado la implementación de nuevas políticas públicas. En este contexto, promover una mayor comprensión sobre su naturaleza, resulta fundamental.

Las interpretaciones tradicionales asocian el fenómeno a mercados segmentados o duales, en los cuales existen barreras de acceso al empleo formal, se trabaja al margen de la regulación, y los trabajadores registran menos ingresos y carecen de prestaciones de seguridad social. En tanto, explicaciones más recientes, sostienen que existen decisiones voluntarias en el ejercicio de un trabajo informal. La presencia de ambas expresiones constituye un tipo de heterogeneidad que es el objetivo de esta investigación.

El problema inicial al cual se enfrenta un investigador es que el concepto de informalidad es amplio y difuso. En un principio, se concibió como una actividad económica precaria, desarrollada al margen de cumplimiento total o parcial de la normativa que la regulaba, pero, gradualmente se han ido incorporando actividades económicas más sofisticadas, que incluyen trabajadores asalariados y a un grupo creciente de trabajadores por cuenta propia. Este último grupo es particularmente interesante, pues ha ido registrando un incremento de su proporción dentro de la fuerza de trabajo, además, comprende desde yacimientos de empleo precario, hasta nuevas formas de empleo basadas en nuevas plataformas tecnológicas que aún no se encuentran debidamente reguladas.

Todas estas actividades tienen en común el incumplimiento total o parcial de regulaciones tributarias, sanitarias o ambientales; y desde el punto de vista de la normativa laboral y previsional, las personas que ejercen estas actividades no cuentan con un vínculo contractual, ni realizan aportes previsionales que les aseguren cobertura de salud o permita obtener en el futuro una pensión.

Coyunturalmente, las mediciones de empleo registran una sistemática disminución del empleo asalariado, en especial el de mayor calidad, y simultáneamente se ha producido un incremento de ocupaciones autónomas (trabajos por cuenta propia). En el período eneromarzo de 2017, la encuesta de empleo (INE, 2017) registra la destrucción de 25 mil puestos de trabajo formales, y se registra la “creación" de 109 
mil trabajos independientes. Este último factor ha permitido mantener una baja tasa de desocupación, pero subyacen en esta cifra cambios más profundos dentro del mercado laboral.

\section{Enfoques teóricos}

A comienzos de los años setenta, Keith Hart desarrolló el concepto de economía informal, aplicado en el marco de un estudio encomendado por la OIT sobre las economías de Kenia (Organización Internacional del Trabajo, 1972) y Ghana (Hart, 1973). Mediante este concepto, se refería al surgimiento de un grupo de trabajadores y pequeñas empresas que operaban fuera del marco principal de la economía formal, afirmaba que "la economía informal es una economía marginal, separada y no conectada directamente con la economía formal que les provee de ingresos o una seguridad a los pobres" (1973). Desde ese entonces, se le conceptualizó como un trabajo precario, y en base a ello, las principales corrientes teóricas, la institucionalista y la estructuralista, se relacionan con esta concepción.

La teoría institucionalista o dualista se concentra en el estudio de las organizaciones informales, su estructura interna, y en su interacción con otras organizaciones. Plantea la existencia de una estructura económica de corte dual, conformada por un sector moderno, y un sector informal. El primero, comprende todas aquellas actividades económicas en las que existen economías de escala en capital humano y físico y, el segundo, se caracteriza por ser bajo en niveles de capacitación y uso de capital físico. Dentro de sus principales promotores se cuenta Tokman (1976), Presbisch (1978), entre otros, pertenecientes al Programa Regional del Empleo de Latinoamérica y el Caribe, citados en PREALC (1978).

Otra corriente relevante es la estructuralista, que sostiene que la estructura económica incide en el mercado de trabajo por la falta de correspondencia cuantitativa y cualitativa entre la oferta y la demanda de trabajo, entendiéndose como una "insuficiencia dinámica" e imperfecciones de la economía formal para crear puestos de trabajo y para capacitar la mano de obra en el sector moderno. Esto implica que, por opción o exclusión, las personas crean su propia fuente de trabajo, entre quienes sostienen este enfoque, se destacan las aportaciones de Alejandro Portes, Manuel Castells, Lauren Benton y Bryan Roberts (1989), entre otros.

Luego, se incorpora la corriente legalista, cuyo principal exponente, Hernaldo De Soto (1989) quien plantea que el origen de la 
informalidad radica en un exceso de regulación por parte del sector público y el exceso de burocracia que hacen de la participación en el sector formal una opción más onerosa, con sectores regidos por normas y regulaciones que exceden el marco normativo socialmente aceptado.

No obstante, en los últimos años, se ha ido desarrollando una visión de corte integradora, mediante la cual, un elemento relevante lo constituye la voluntariedad al momento de la decisión de participar en el sector informal, aspecto tratado por distintos autores; (Hart, 1973), (Organización Internacional del Trabajo, 1972), (Maloney, 2002), (Fields, 2005).

Se configura una interpretación integradora, que habla del "escape" como una decisión "adoptada por opción entre quienes prefieren una actividad más flexible, autónoma y en algunos casos con mejores ingresos porque, a diferencia de los trabajos formales, no pagan impuestos, ni contribuyen a regímenes de protección social" (Fields, 2005), (Perry y otros, 2007).

Esta interpretación remarca la presencia de dos tipos distintos de trabajadores informales: los excluidos, y los que optan por la informalidad (Pagés-Serra y Madrigal, 2008), afirmando que muchos trabajadores, empresas y familias escogen su nivel óptimo de adherencia a los mandatos y las instituciones del Estado, lo que depende del valor que asignen a los beneficios netos relacionados con la formalidad y al esfuerzo y la capacidad de fiscalización del Estado. Es decir, realizan análisis implícitos de costo-beneficio acerca de si deben o no cruzar el margen hacia la formalidad, y con frecuencia, deciden no cruzarlo (Perry y otros, 2007).

La posibilidad que los agentes se encuentren ejerciendo la elección del tipo de actividad laboral, sucede en un mercado que funciona bajo condiciones integradas (no segmentadas) y competitivas. En él, la decisión del individuo pasa por su interés de maximizar sus utilidades e ingresos, optando en este caso por los mayores ingresos obtenidos dentro del mercado informal que los que podría obtener formalmente (Maloney W., 1999).

En este contexto, se afirma que los trabajadores informales conforman su propio dualismo o heterogeneidad, al estar compuestos por grupos de personas que representan una combinación de alternativas voluntarias y obligatorias (Fields, 2005), (Günther y Launov, 2006), hipótesis explorada dentro de la investigación realizada.

Para una posterior formulación del modelo, se ha recopilado referencias bibliográficas que describen o justifican la relación de atributos determinantes sobre la informalidad laboral, y en general, se 
constata que no existe consenso respecto a cuáles son estos factores (Maloney, 2002), lo que en parte es consecuencia de las diversas interpretaciones conceptuales que persisten hasta la fecha.

En la literatura sobre esta temática, se encuentran referencias a determinantes del trabajo informal según las unidades de análisis que estén siendo aplicadas: unidades económicas (OIT,1993 y 1999), personas (Tokman, 2004); categorías ocupacionales (PREALC, 1978); hogares (Villagómez, 1999); capital, tecnología, productividad e ingresos (Levenson y Maloney, 1998), (Maloney, 2002). La heterogeneidad en las formas de medición se ve reflejada en las múltiples definiciones alternativas existentes.

\section{Fuente de datos}

Las encuestas de hogares y las de empleo constituyen las principales fuentes de datos utilizados para el estudio de la informalidad en Latinoamérica. En Chile, a comienzos del año 2018, se incorporaron preguntas específicas dentro de la Encuesta Nacional de Empleo que han permitido iniciar la publicación regular de estadísticas sobre informalidad laboral, las que constituirán una referencia central para futuras investigaciones.

Esta investigación se inició cuando se publicaron los resultados de la Encuesta de Caracterización Socioeconómica CASEN (Ministerio de Desarrollo Social, 2016) con datos obtenidos en el año 2015. Esta encuesta contiene datos de ocupación, ingresos (del trabajo y otros), estructuras de los hogares, vivienda, educación y salud.

El principal motivo para elegir esta fuente de datos es la disponibilidad de información sobre ingresos formales e informales, los cuales se encuentran vinculados de manera innominada con una serie de características de los individuos y de sus hogares, un tipo de información que adolecen las encuestas de empleo.

Además, es posible utilizar las encuestas aplicadas de manera bianual desde el año 2009 en adelante, fecha en que se introduce un ajuste al cuestionario con la finalidad de capturar de mejor modo información sobre ingresos de actividades no formales e ingresos formales de los autónomos. 


\section{Informalidad laboral y estructura del mercado de trabajo}

Chile es un país con una población estimada en el año 2015 de 18.128.266 personas. De ellas, 14.498 .600 se encuentran en edad de trabajar, y 8.019.076 (un 55.3 por ciento) forman parte de la fuerza de trabajo.

Para la selección de una herramienta de medición, se recurre a una caracterización del mercado de trabajo, donde se revisan tres dimensiones principales: antecedentes sobre la estructura del empleo (asalariados y autónomos); la relación del empleo con las principales coberturas de seguridad social, y el trabajo en pequeñas unidades económicas. Del análisis realizado, se concluye que los más relevantes son: la estructura del empleo, las categorías ocupacionales y la información de cumplimiento total o parcial de la regulación laboral o tributaria. Estos elementos constituyen la base para el diseño de un tipo medición del trabajo informal especialmente elegido para esta investigación.

La principal categoría de empleo la constituye la población asalariada, con 5.366.339 personas en el año 2015. Dentro de ella, el principal atributo para definir la formalidad de una relación laboral es la existencia de un contrato de trabajo escrito, instrumento que detalla la función a desempeñar, remuneraciones, beneficios y los aportes para protección social (cobertura sanitaria, de pensiones, de salud ocupacional y desempleo). Además, permite asegurar el cumplimiento de regulaciones en materia de salario mínimo, extensión de las jornadas de trabajo y otras normas especiales que regulan la actividad laboral.

Sobre esta categoría ocupacional, es importante destacar que durante la última década se llegó a registrar un 25 por ciento de personas asalariadas que declaraban trabajar sin contrato escrito, cifra que ha logrado reducirse en 2015 a un 13.8 por ciento. Sin embargo, sigue siendo una magnitud importante, pues cerca de 700.000 personas declaran no contar con este vínculo formal mientras reconocen el cumplimiento de subordinación y dependencia laboral de un tercero.

La segunda categoría en orden de importancia la constituye el trabajo autónomo, constituido por trabajadores por cuenta propia (1.411.452 personas) y empleadores (145.614), los que corresponden a un 11.6 por ciento de la fuerza de trabajo y a un 23.2 por ciento de la fuerza de trabajo ocupada. 
En este caso, la clasificación como trabajo formal se encuentra asociada al cumplimiento de la regulación tributaria. En la encuesta de hogares del año 2015, un 10,7 por ciento de esta categoría de empleo autónomo a prestaciones de servicios (responden que emiten boletas de honorarios) y un 17,1 por ciento registran ventas y emiten boletas de compraventa. Es decir, 3 de cada 10 personas de esta categoría cumplen con la regulación vigente, y por este motivo pueden ser clasificados como trabajadores formales.

En Chile, el trabajo autónomo aún se encuentra eximido de realizar pagos obligatorios para un seguro sanitario, de salud ocupacional, de cesantía y ahorro previsional. Esta particularidad produce una diferencia entre ingresos brutos y netos, producto de los pagos previsionales que alcanzan aproximadamente a un 26 por ciento de los ingresos brutos.

Si bien, la incorporación de los autónomos a la obligatoriedad de pagar cotizaciones fue establecido en la reforma del sistema de pensiones del año 2008, su entrada en vigor ha sido postergada en dos ocasiones desde su promulgación. Cuando comience su aplicación, se igualará la obligatoriedad de hacer pago de cotizaciones previsionales a todos quienes desempeñen actividades laborales remuneradas.

Los efectos de haber aplicado durante décadas este esquema de voluntariedad en los autónomos, continuará repercutiendo sobre los montos de ahorro acumulados por parte de quienes han trabajado bajo esta modalidad durante su trayectoria laboral.

Finalmente, es conveniente consignar que un trabajador formal también puede extender la cobertura de su seguro de salud (sanitario) a su grupo familiar más directo: esposa, sin seguro propio, e hijos (menores de 18 años; 24 si se encuentran estudiando). Esta extensión de cobertura familiar constituye un desincentivo para la formalización de mujeres y jóvenes. (IPS y FLACSO, 2017).

\section{Medición de la informalidad laboral}

Producto que existen múltiples enfoques teóricos y alternativas de medición, es relevante la definición de informalidad que se utilice, al respecto, la Oficina Internacional del Trabajo ha propiciado la articulación y convergencia conceptual en torno a una definición general, la cual ha sido discutida y consensuada en Conferencias Internacionales de Estadísticos del Trabajo (Organización Internacional del Trabajo, 2003, 2012). 
Los datos disponibles permiten contar con información sobre ingresos y características del individuo y de su grupo hogar: diferencias territoriales (urbano/rural), tamaño de empresa, estructura de grupo familiar, nivel educacional, experiencia, género y edad.

Se clasifican los datos en base a categorías ocupacionales y a requisitos de cumplimiento de la normativa laboral o tributaria que impone la legislación chilena a cada categoría ocupacional. Los mismos se detallan a continuación:

- En los trabajos asalariados (incluye a empleos privados, públicos y trabajadoras domésticas) se considera que una actividad es formal cuando existe un contrato de trabajo escrito y firmado.

- En los trabajos autónomos (de empleadores y trabajadores por cuenta propia) se considera como trabajo formal a quienes emiten boleta de prestación de servicio o de compraventa, esto implica el cumplimiento de nuestra legislación tributaria. Este dato es posible obtenerlo en las encuestas de hogares aplicadas desde 2009 en adelante.

- En los trabajos de familiares no remunerados, por definición, se les considera una categoría de trabajo informal.

Esta fórmula, para efectos de la presente investigación, será denominada medición de Informalidad Laboral. Como resultado, se estima que en el año 2015 un total de 1.974 .770 personas trabajó bajo esta modalidad, lo que equivale a un 26,2 por ciento de la población ocupada. Se registra una mayor proporción de hombres, 1.107.024, que supera al número de mujeres estimado en 867.746. Es decir, en términos absolutos, el segmento informal reproduce y contribuye con una mayor proporción también en la participación laboral "informal" masculina.

Lo interesante es que, contrario a lo que se señala comúnmente, sólo un 60 por ciento corresponde a trabajadores autónomos y familiares no remunerados, que suelen ser el foco de atención, sino que también está compuesto en un 40 por ciento de trabajadores asalariados sin contrato.

En cuanto a la distribución por grupos socioeconómicos, al segmentar por quintiles socioeconómicos, se confirma una mayor presencia de informalidad laboral entre las personas de menores ingresos. Mientras que, en el primer quintil socioeconómico, la proporción de estos trabajadores alcanza a un 49,6 por ciento, en el quinto, llega sólo a un 15,3 por ciento. 
Gráfico 1: Distribución de trabajadores Formales e Informales clasificados por quintiles Socioeconómicos y por Sexo 2015. Fuente: Elaboración en base a Encuesta de Hogar CASEN 2015, MDS (2016)

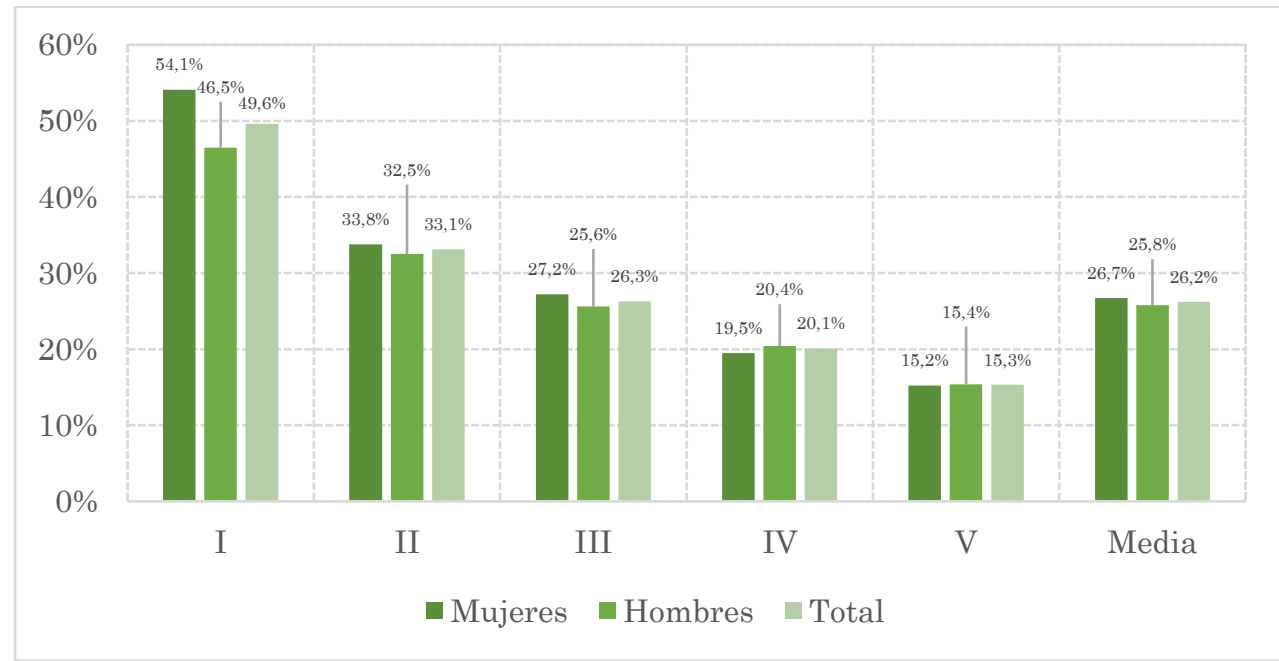

Gráfico 2: Tasa de Informalidad Laboral por Categoría Ocupacional y Sexo, 2015. Fuente: Elaboración en base a Encuesta de Hogar CASEN 2015, MDS (2016)

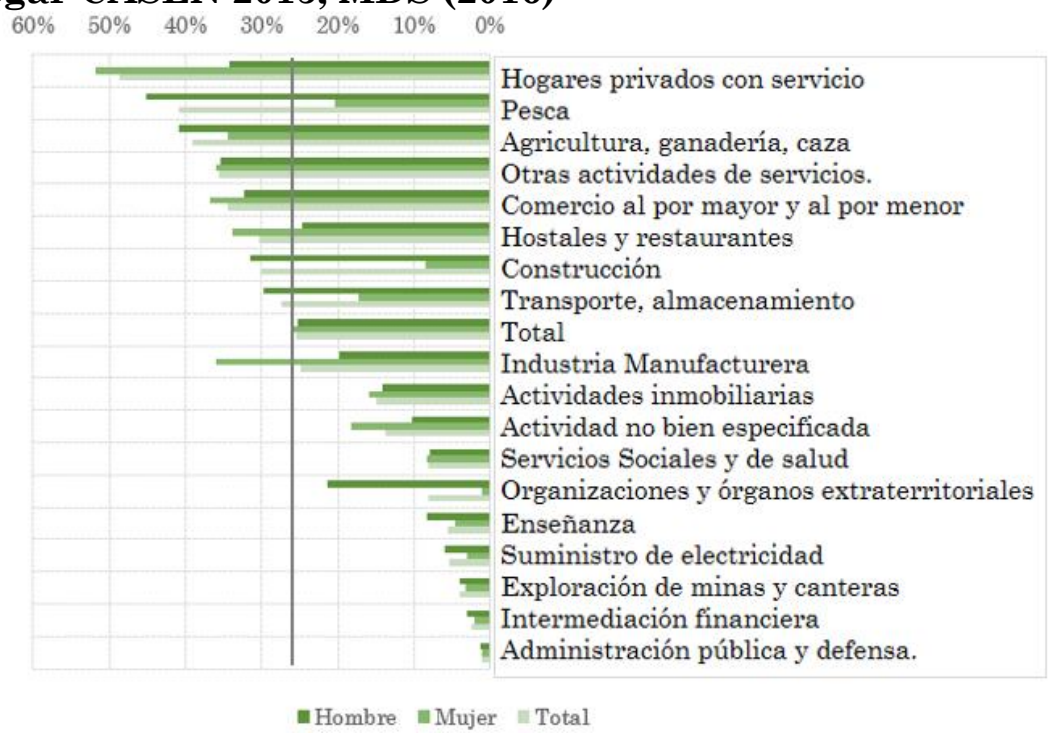


Respecto de las ramas de actividad económica que concentran una mayor proporción de trabajadores informales ocupados, éstas corresponden a industrias extractivas (pesca y agricultura), rubro de construcción, y servicios (hoteles, comercio, otras actividades de servicio).

Por el contrario, las actividades que concentran una mayor proporción de trabajos formales son aquéllas vinculadas a la actividad pública (sector público, salud, enseñanza), sectores regulados (electricidad, gas y agua, intermediación financiera) y sectores con alta regulación de salud y seguridad ocupacional, como el sector minero.

\section{Variación de la informalidad laboral y previsional}

Uno de los argumentos esgrimidos al momento de formular esta investigación fue la necesidad de definir un método de medición dentro del amplio espectro de posibilidades. Por este motivo se ha optado por la utilización de un método de medición de informalidad laboral que ha sido definida en la sección anterior.

Durante el proceso de búsqueda de fórmulas de medición se identificó una definición alternativa, que denominaremos "informalidad previsional", la cual define como trabajos formales a aquellos empleos capaces de generar acceso y cobertura a esquemas de protección social ${ }^{1}$. Sin embargo, esta opción no fue adoptada como instrumento de medición debido a la particular estructura de seguridad social existente en Chile, especialmente, por el efecto de la voluntariedad de la cotización de los trabajadores autónomos.

Bajo este esquema contributivo, quienes se ocupan en ventas de bienes y/o servicios cumplen total o parcialmente con los requisitos de formalidad que establece la autoridad tributaria (Servicio de Impuestos Internos), mientras que, de manera simultánea no cumplen con el criterio de formalidad definido para la informalidad previsional. Mientras se mantenga la voluntariedad en el pago, afectará la aplicación de métodos de medición que se basen la cobertura de seguridad social. A pesar de lo reparos explicitados, si se comparan ambos métodos de clasificación, aplicados sobre los datos de las encuestas CASEN del período 2009 a

\footnotetext{
1 Existen numerosas referencias al uso de esta modalidad de clasificación, entre ellas, destacan la Conferencia Internacionales de Estadísticos del Trabajo (CIET - OIT, 1993) y autores como Fields (2005), Perry y otros (2007).
} 
$2015^{2}$, se constata en el gráfico 3 que se obtienen resultados divergentes entre ambas mediciones.

\section{Gráfico 3: Evolución Tasa de Informalidad Laboral y Previsional 2009 - 2015. Fuente: Elaboración en base a Encuesta de Hogar CASEN 2015, MDS (2016)}

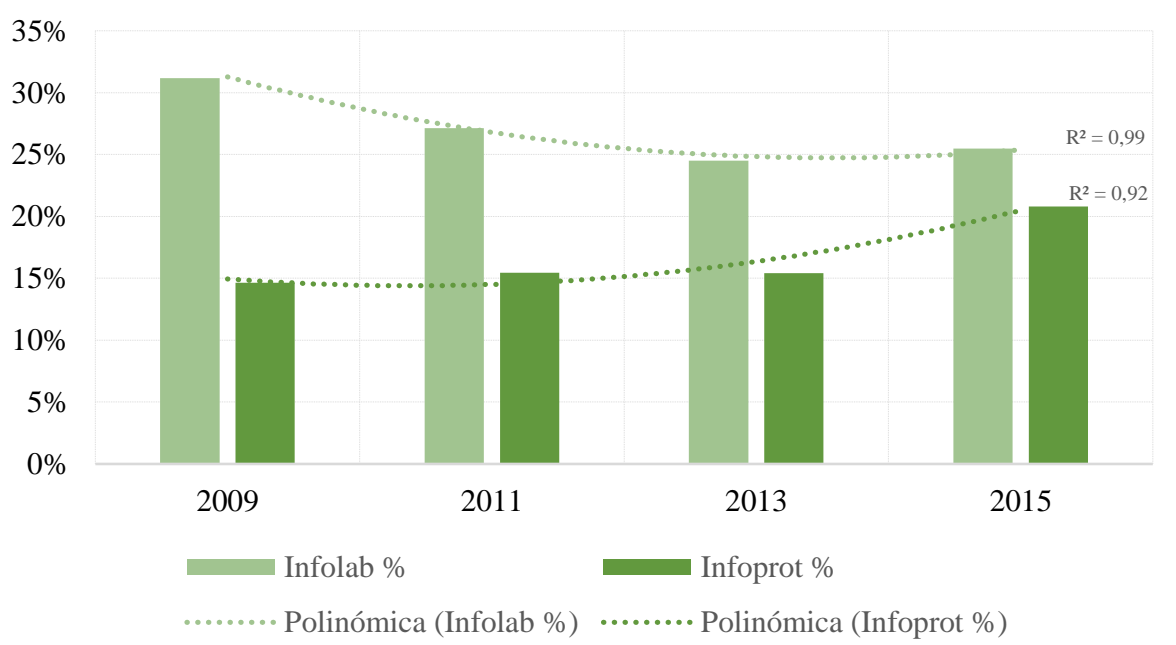

En esta comparación se evidencia los efectos del deterioro del empleo asalariado y un simultáneo incremento de empleos autónomos. En el año 2009, mientras la medición basada en protección social registra una tasa de un 15 por ciento de la población ocupada, la informalidad laboral alcanza un 30 por ciento.

Luego, al observar su evolución hacia 2015, la medición laboral en 2015 ha registrado una disminución de casi cuatro puntos porcentuales respecto al año 2009, y en ese mismo período, la alternativa previsional registra en este mismo período un aumento de más de cinco puntos porcentuales.

Lo anterior confirma que, si bien, ambas mediciones representan fenómenos relacionados, su disímil comportamiento confirma la relevancia y no trivialidad de la forma de medición de la informalidad. La comparación de estas dos mediciones, ambas aplicadas a los mismos

2 Se utilizan los datos de encuestas CASEN desde 2009, año en que se incorpora una segunda pregunta sobre la realización de actividad remunerada que permite capturar actividades informales u ocasionales a cambio de un sueldo o salario. 
León Ossandón / Revista de Economía Laboral 15(2) (2018), 57-89

datos chilenos puede llevar a conclusiones diferentes, y permite confirmar que el fenómeno en estudio se encuentra representado de manera adecuada utilizando la métrica de informalidad laboral elegida.

\section{Formulación de modelo de estimación con heterogeneidad esencial}

Una vez que se ha seleccionado una forma de medición, y que se dispone de una fuente de datos adecuada, se formula la pregunta inicial de esta investigación: ¿Es el trabajo informal una opción voluntaria o corresponde a una condición obligada?

Con el fin de contestar a la pregunta guía de la investigación, se aplica una técnica de variables instrumentales a un modelo de resultados y opciones de tratamiento con presencia de heterogeneidad esencial.

Este tipo de modelo ha sido propuesto por Heckman, Urzúa y Vytlacil (2006) quienes se centran en la interacción entre heterogeneidad y auto - selección. Ésta se presenta cuando en un modelo las respuestas a las intervenciones son heterogéneas y los agentes adoptan el tratamiento con conocimiento al menos parcial de su respuesta idiosincrásica y presenta evidencia de que existe autoselección del tratamiento (Arcand y Bassole, 2011).

La adaptación de este modelo a la economía laboral y al estudio de la informalidad es un aporte de Natalia Radchenko $(2014,2016)$ quien ha asimilado el concepto de tratamiento al de una elección de trabajo formal y la presencia de heterogeneidad esencial.

En este contexto, se permite inferir que los agentes se encuentran eligiendo en función de consideraciones de coste/beneficio respecto de si participan de manera formal o informal en el mercado de trabajo.

En Heckman y Vytlacil (2005) y Heckman, Urzúa y Vytlacil (2006) se establece que el estimador más adecuado para relacionar un resultado con las características de un individuo es el Efecto Marginal del Tratamiento (MTE, Marginal Treatment Effect), que describe el cambio marginal, dado un deseo no observado de participar del tratamiento. Su elección aventaja otros estimadores, debido a que estos últimos pueden ser obtenidos como promedios ponderados de éste. (Vytlacil, 2013).

Para su cálculo, se utiliza el método de Emparejamiento por Puntaje de Propensión (Propensity Score Matching) ${ }^{3}$, introducido por Rosenbaum y Rubin (citado en Zhou X, Xie Y, 2016), el cual permite

\footnotetext{
${ }^{3}$ Cálculos son realizados mediante el uso del software Stata.
} 
reconstruir un hipotético proceso de aleatorización no llevado a cabo debido a la clasificación aplicada sobre la muestra. Según estos autores, el uso del MTE funciona mejor cuando hay una variable instrumental válida y fuerte (IV).

Este modelo es un caso particular, en donde el tratamiento tiene sólo dos estatus: Tratado (1), No tratado (0) y dos resultados posibles: el ingreso mensual generado por un trabajo formal (ln $W_{1 i}$ ) y el generado de manera informal (ln $W_{0 i}$ ), ambos expresados en base logarítmica. Tal como lo define Radchenko (2014):

$$
\begin{aligned}
& \ln W_{1 i}=\alpha_{1}+\beta_{1} X_{i}+U_{1 i} \\
& \ln W_{0 i}=\alpha_{0}+\beta_{0} X_{i}+U_{0 i}
\end{aligned}
$$

$\alpha_{0}, \alpha_{1}, \beta_{0} y \beta_{1}$ son los parámetros desconocidos de las ecuaciones salariales, $\mathrm{X}_{\mathrm{i}}$ son las características observadas y $\mathrm{U}_{1 i}$ y $\mathrm{U}_{0 i}$ las no observadas y $E\left(U_{1 i} \mid X_{i}\right)=0$ y $E\left(U_{0 i} \mid X_{i}=0\right)$

Para el cálculo de un estimador, el problema fundamental de la inferencia causal se encuentra en la dificultad para realizar estimaciones donde se observa solamente $\ln W_{1 i}$ o $\ln W_{0 i}$, y no ambos, para cada persona (Wooldridge, 2004) (Zhou y Yu, 2016). Dado que ambos estados no pueden ser observados simultáneamente, este sistema puede expresarse como una combinación de ambas ecuaciones:

$$
\ln W_{i}=\alpha_{0}+\beta_{0} X_{i}+\Delta_{i} D_{i}+U_{0 i}
$$

donde el efecto del tratamiento individual $\Delta$ es:

$$
\Delta_{i}=\left(\alpha_{1}-\alpha_{0}\right)+\left(\beta_{1}-\beta_{0}\right) X_{i}+\left(U_{1 i}-U_{0 i}\right)
$$

El primer componente de esta ecuación, $\left(\alpha_{1}-\alpha_{0}\right)$, corresponde a la brecha homogénea existente entre los no tratados y tratados; luego, el segundo componente $\left(\beta_{1}-\beta_{0}\right) X_{i}$, da cuenta de las diferencias producto de diferencias relacionadas con las características observables; y finalmente, un tercer componente $\left(U_{1 i}-U_{0 i}\right)$ se relaciona con la heterogeneidad esencial, relacionado con los heterogéneos retornos en ingresos (de ejercer un trabajo formal respecto de ejercer uno informal), producto a determinantes salariales no observados.

El componente de participación en sector formal viene dado por: 


$$
D_{i}=\left\{\begin{array}{r}
1 \text { si } I_{i}>0 \\
0 \text { si } I_{i} \leq 0
\end{array}\right.
$$

donde $I_{i}=\gamma Z_{i}$ - $V_{i}$, I corresponde a la función la probabilidad de trabajar de manera formal, obtenida mediante del Puntaje de Propensión. Z corresponde a un set de instrumentos para D.

Dado que las características observadas $Z_{i}$ y $V_{i}$ y las variables no observadas $\left(U_{1 i}\right.$ y $\left.U_{0 i}\right)$ y $V_{i}$ se encuentran correlacionadas. Es decir, los factores no explicados de optar por el trabajo formal tienen relación con los factores no explicados en la determinación de los ingresos.

Se cumple que:

$$
\mathrm{P}\left(Z_{i}\right)=P\left(\gamma Z_{i-} V_{i}>0\right)
$$

$\mathrm{Z}$ afecta la condición de tratamiento sólo a través de los puntajes de propensión $\mathrm{P}(\mathrm{Z})$. (Zhou y $\mathrm{Yu}, 2016$ ). Por la propiedad de transformación integral, y sin pérdida de generalidad, $\mathrm{U}_{\mathrm{D}}$ es definida como una variable aleatoria uniforme (Heckman, 2007) por construcción, puede normalizarse arbitrariamente el término de error en la ecuación de selección $V_{i}$, distribuyéndolo según una densidad uniforme dentro de un intervalo [0,1], lo que implica que el tratamiento se produce cuando $y Z_{i}>U D$. (Arcand y Bassole, 2011) (Heckman y Vytlacil, 2005).

Para el individuo $\boldsymbol{i}$ no es observable, pero puede ser calculada en el punto en donde el trabajador es indiferente a participar entre el segmento formal o informal. En él: $V_{i}=\gamma Z_{i} \mathrm{y}$, al aplicar sobre esta expresión la función de densidad acumulativa normal $F_{V}$, se obtiene $F_{V}$ $\left(\gamma Z_{i}\right)=F_{V}\left(V_{i}\right){ }^{4}$

En este punto, se obtiene el Efecto Marginal del Tratamiento (MTE), puede ser igualado a la función del Puntaje de Propensión $P(Z)$, que representa la probabilidad de selección del trabajo formal, estando condicionado a las variables observadas $X$ que se encuentran fijas en un valor $\mathrm{x}$, y las no observables $V$, en un valor v, y p es un valor de evaluación particular del puntaje de propensión.

$$
\begin{aligned}
\operatorname{MTE}\left(\mathrm{x}, \mathrm{U}_{\mathrm{D}}\right) & =\frac{\partial\left(\ln W_{i} \mid X_{i}=x_{i}, P(Z)=p\right)}{\partial p} \\
\operatorname{MTE}(\mathrm{x}, \mathrm{v}) & =\mathrm{E}\left(\ln W_{1}-\ln W_{0} \quad \mid \mathrm{X}=\mathrm{x}, \mathrm{V}=\mathrm{v}\right) \\
& =\mathrm{E}\left(\ln W_{1}-\ln W_{0} \quad \mid \mathrm{X}=\mathrm{x}, \mathrm{U}_{\mathrm{D}}=\mathrm{uD}=\mathrm{P}(\mathrm{Z})=\mathrm{p}\right)
\end{aligned}
$$

\footnotetext{
${ }^{4}$ En términos de $U_{D}$ puede notarse: $\mathrm{U}_{\mathrm{Di}}=\mathrm{F}(\gamma \mathrm{Zi})$.
} 
De este modo, la predicción de los Puntajes de Propensión permite definir los valores de $U_{D}$ sobre los cuales el MTE puede ser identificado.

Se hace uso de la clasificación propuesta por el trabajo, lo que constituye un diseño no aleatorizado, por lo tanto, debe verificarse el cumplimiento una serie de condiciones o supuestos previos para la especificación del modelo.

Se debe verificar y escoger datos que cumplan con los siguientes supuestos previos:

- Independencia de Medias Condicionadas. (CMI), supuesto que restringe la dependencia del modelo de tratamiento y sus potenciales resultados. Al asumir el condicionamiento de covariables, se puede obtener un resultado de similar calidad a los que se obtiene con una asignación aleatoria. (Heckman, Urzúa y Vytlacil, 2006).

- Superposición o soporte Común, supuesto que asegura que cada individuo pueda tener una probabilidad positiva de recibir cualquier nivel de tratamiento. La evaluación del o los estimadores se aplica sólo sobre las submuestras que forman parte de la superposición.

- Muestra independiente e idénticamente distribuida (IID).

Rosenbaum y Rubin (1983) según lo citado en Wooldridge (2009) denominan a la combinación de los dos primeros supuestos ignorabilidad fuerte. Este supuesto, aplicado como base de los métodos basados en puntajes de propensión, establece que, a través del control de un set definido de covariables, se supone que el estatus del tratamiento es un elemento independiente de los potenciales resultados del modelo.

\section{Estimación del efecto marginal del tratamiento}

Para la formulación del modelo e hipótesis, se utiliza como variable observada de resultados los ingresos mensuales de las personas, los cuales son obtenidos de datos provenientes de la Encuesta de Caracterización Socioeconómica CASEN. La información incluye los ingresos de la ocupación principal, y también los de una ocupación secundaria (en caso de que el encuestado lo informe). Asimismo, incluye 
los ingresos generados por la auto-provisión de bienes producidos por el hogar $^{5}$.

Se aplica la definición de informalidad laboral adoptada y se realiza la clasificación entre trabajadores formales e informales a los datos provenientes del set de datos.

La relación existente entre los ingresos personales y las variables observadas (vector X), se plasma en una Ecuación de Mincer (1958), para la cual se utilizan: experiencia laboral, horas trabajadas, escolaridad, valor cuadrático de escolaridad, edad, valor cuadrático de edad, tamaño de empresa, sexo y zona (urbano/rural). El resultado se muestra en el cuadro 1.

Luego, utilizando una regresión tipo Probit, se estiman los Puntajes de Propensión asociados a la probabilidad condicional de recibir el tratamiento, dadas las características observadas. Por convención, se asigna como tratado a quien ejerce el trabajo formal $(\mathrm{D}=1)$ y como no tratados al del trabajo informal $(\mathrm{D}=0)$, se asume como supuesto que las variables instrumentales de $\mathrm{Z}$ son independientes del tratamiento, y que existe linealidad de los errores $\mathrm{U}$ en $\mathrm{X}$.

Las variables utilizadas para estimar la probabilidad de trabajo formal mediante el cálculo de un puntaje de propensión (vector Z), también contribuyen a la determinación de los salarios (vector X), por lo que es necesario diferenciar ambos sets de variables, de modo tal que se pueda capturar el efecto de variables no observadas. Para ello, se construye e introduce en $\mathrm{Z}$ una variable que contabiliza el número de trabajadores informales existentes dentro del grupo familiar, usando la definición de formalidad elegida y la estructura de los hogares que provee la encuesta.

Esta variable instrumental tiene el propósito de contribuir a la disminución de la varianza en la estimación y su principal atributo es que incide en la probabilidad de adoptar el tratamiento (trabajar formalmente), pues las características del hogar inciden en la forma de trabajo; y simultáneamente, no incide sobre la determinación del salario.

Antes de iniciar la estimación, se aplican los supuestos previos detallados en el punto precedente, y como principal resultado, se obtiene que la muestra que cumple el criterio del soporte común o superposición reduce a un 40,7 por ciento los datos originales.

5 La encuesta incorpora una pregunta complementaria al registro de actividades remuneradas, lo que permite capturar los ingresos que generan las actividades informales u ocasionales: a cambio de un sueldo o salario, ingresos por su cuenta, en una empresa, en especies o en metálico, como aprendiz, o que haya realizado una actividad para un familiar. 
Cuadro 1: Relación de Ingresos y Coeficientes estimados para trabajo informal $\beta_{0}$, brecha trabajo formal-informal $\left(\beta_{1-} \beta_{0}\right)$ año 2015. Fuente: Elaboración en base a Encuesta de Hogar CASEN 2015, MDS (2016)

\begin{tabular}{|c|c|c|}
\hline Características de Trabajadores & $\begin{array}{c}\text { Retorno de } \\
\text { empleo informal } \\
B_{0}(\mathrm{SE}) \\
\end{array}$ & $\begin{array}{c}\text { Brecha de Retorno } \\
\text { Coeficiente formal-informal } \\
\left(B_{1}-B_{0}\right)(\mathrm{SE})\end{array}$ \\
\hline \multirow[t]{2}{*}{ Horas Trabajadas } & 0,0176 & $-0,0068$ \\
\hline & $-0,0003$ & 0,0000 \\
\hline \multirow[t]{2}{*}{ Escolaridad } & $-0,0363$ & $-0,0625$ \\
\hline & $-0,0042$ & 0,0000 \\
\hline \multirow[t]{2}{*}{ Escolaridad Cuadrática } & 0,0057 & 0,0031 \\
\hline & $-0,0002$ & 0,0000 \\
\hline \multirow[t]{2}{*}{ Edad } & 0,0496 & $-0,0081$ \\
\hline & $-0,0017$ & 0,0000 \\
\hline \multirow[t]{2}{*}{ Edad Cuadrática } & $-0,0005$ & 0,0001 \\
\hline & $-0,0000$ & 0,0000 \\
\hline \multirow[t]{2}{*}{ Experiencia } & 0,0043 & 0,0032 \\
\hline & 0,0004 & 0,0000 \\
\hline \multicolumn{3}{|l|}{ Tamaño de Empresa } \\
\hline \multirow[t]{2}{*}{ Sólo una persona } & $-0,2796$ & 0,0900 \\
\hline & $-0,0218$ & $-0,0006$ \\
\hline \multirow[t]{2}{*}{ de 2 a 5 personas } & $-0,0624$ & $-0,0770$ \\
\hline & $-0,0226$ & $-0,0006$ \\
\hline \multirow[t]{2}{*}{ de 6 a 9 personas } & $-0,0702$ & 0,0366 \\
\hline & $-0,0303$ & $-0,0010$ \\
\hline \multirow[t]{2}{*}{ de 6 a 9 personas } & $-0,0007$ & $-0,0141$ \\
\hline & $-0,0264$ & $-0,0008$ \\
\hline \multirow[t]{2}{*}{ de 50 a 199 personas } & $-0,0198$ & 0,0606 \\
\hline & $-0,0315$ & $-0,0011$ \\
\hline \multirow[t]{2}{*}{200 y más personas } & 0,0578 & 0,0261 \\
\hline & $-0,0299$ & $-0,0009$ \\
\hline \multirow[t]{2}{*}{ Sexo /Mujer (Relativo a hombres) } & $-0,3458$ & 0,1013 \\
\hline & $-0,0092$ & $-0,0001$ \\
\hline \multirow[t]{2}{*}{ Zona rural (relativo a urbano) } & $-0,1692$ & 0,0887 \\
\hline & $-0,0123$ & $-0,0002$ \\
\hline \multirow[t]{2}{*}{ Constante } & 10,3892 & 0,8756 \\
\hline & $-0,0454$ & $-0,0026$ \\
\hline
\end{tabular}

Para la obtención de los Puntajes de Propensión, se aplica una regresión del tipo PROBIT. Al graficar de manera separada las submuestras de trabajadores formales e informalidad, se obtiene el gráfico 4. 
Gráfico 4: Puntajes de Propensión para Trabajadores Formales e Informales. Fuente: Elaboración en base a Encuesta de Hogar CASEN 2015, MDS (2016).

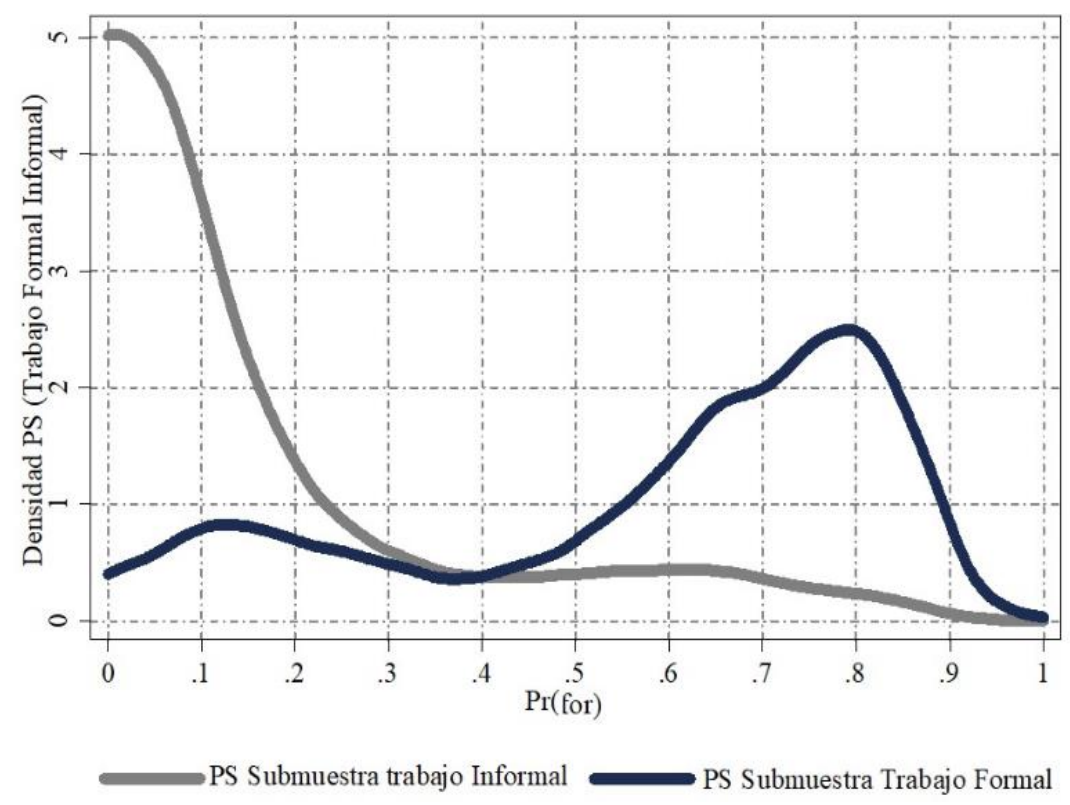

Dentro de la submuestra de trabajadores informales, como es de esperar, la densidad de probabilidades se concentra en torno del sector izquierdo de la gráfica. Esto significa que una mayoría de los trabajadores informales tienen una alta probabilidad de mantenerse en la informalidad. La media de este grupo se sitúa en un 18 por ciento y, sólo un 11 por ciento de los casos excede un cincuenta por ciento de probabilidad de obtener trabajo formal.

Por el contrario, dentro de la submuestra de los trabajadores que actualmente desempeñan una actividad formal, se obtiene una media de 60 por ciento de probabilidad de mantenerse como tales, y un 34 por ciento de ellos tiene una probabilidad inferior a un cincuenta por ciento de ejercer un trabajo informal.

\section{Formulación de hipótesis}

La hipótesis que se desea probar es la presencia de heterogeneidad esencial en la selección de un trabajo formal. Para estos 
efectos se recurre al modelo de efectos de tratamiento, utilizando los fundamentos descritos en las ecuaciones (1) a (6).

En esta base conceptual, se establece que los valores del puntaje de propensión permiten definir los valores para los cuales el estimador puede ser definido, por lo tanto, el estimador puede adoptar la forma de una función no lineal del puntaje de propensión $p$.

En consecuencia, la formulación de la hipótesis puede reducirse a una prueba de linealidad del componente $p$, que corresponde a la función de los puntajes de propensión relacionados con la probabilidad de estar trabajando de manera formal, aplicado al set de datos escogidos. (Heckman, Urzúa y Vytlacil, 2006).

La prueba de linealidad se aplica utilizando el valor esperado de los ingresos, expresados en escala logarítmica, mediante el uso de la relación descrita en la Ecuación (11), en donde se busca determinar si el componente de la función $K(p)$ corresponde a una función no lineal del puntaje de propensión. Los parámetros $\beta_{0} \mathrm{y}\left(\beta_{1-} \beta_{0}\right)$ son estimados a partir de las ecuaciones (1) y (2). ${ }^{6}$

Si se prueba que $K(p)$ es lineal en $p$, esto se debería a que, en ausencia de heterogeneidad esencial, $\beta \perp D$ y $\left(\ln W_{1}-\ln W_{0}\right)$, lo que permiten deducir que la expresión $\mathrm{E}(\ln W \mid P(Z)=p, X=x)$ es lineal en $\mathrm{p}$, implicando que los coeficientes de $\mathrm{p}$ con una potencia igual o superior a dos son estadísticamente no significativos.

Para probar ello, Heckman, Schmierer y Urzúa (2007) proponen un método secuencial simple, que comienza con sólo un término lineal en $p$, y luego, se eleva la potencia sucesivamente, de modo tal que si luego de agregar un término cuadrático, su coeficiente es significativo, no es posible negar la presencia de heterogeneidad esencial.

Manteniendo el condicionamiento en $\mathrm{X}$ implícito, se pueda establecer que se cumple:

$$
\mathrm{E}(\ln W \mid X=x, P(Z)=p)=K(p)
$$

Teniendo en consideración las ecuaciones (3) y (4)

$$
\begin{gathered}
\mathrm{E}(\ln W \mid \mathrm{X}=\mathrm{x}, \mathrm{P}(\mathrm{Z})=\mathrm{p})=\mathrm{E}\left(\ln W_{0} \mid \mathrm{X}=\mathrm{x}, \mathrm{P}=\mathrm{p}\right)+\mathrm{E}\left(\mathrm{D}\left(\ln W_{1}-\ln W_{0}\right) \mid\right. \\
\mathrm{X}=\mathrm{x}, \mathrm{P}=\mathrm{p})
\end{gathered}
$$

Puede ser igualado a la expresión:

\footnotetext{
${ }^{6}$ Coeficientes estimados han sido incluidos en cuadro 1.
} 


$$
\mathrm{E}(\ln W \mid X=x, P(Z)=p)=\alpha_{0}+\beta_{0} x+\left(\left(\beta_{1-} \beta_{0}\right) x\right) p+K(p)
$$

donde la última igualdad, proviene del supuesto de independencia de la variable instrumental, y el supuesto que los errores son lineales en X.

Entonces, la hipótesis se formula de la siguiente manera:

$$
\begin{aligned}
& H_{0}: K(p)=a+b p, \text { para algún } a, b \in R \\
& H_{1:} N_{0} H_{0}
\end{aligned}
$$

La prueba se aplica asumiendo que la no linealidad puede probarse con una estructura polinómica de $p$, condicionada en $X$.

$$
K(p)=\sum_{j=0}^{d} \phi_{j} p^{j}
$$

$\mathrm{H}_{0}: \phi_{j}=0$ para $j=2 \ldots \mathrm{n}$

$\mathrm{H}_{1}$ : No $\mathrm{H}_{0}$

Se utiliza la expresión de la ecuación (10) para y evaluar así la hipótesis de linealidad. Para ello, se estima los coeficientes de p, primero para una expresión lineal, luego se agrega la expresión cuadrática y así sucesivamente, hasta determinar la potencia de la ecuación, basado en la significatividad estadística de los coeficientes.

Los resultados se presentan en el cuadro 2. En este se observa que, a partir del término cuadrático, no es posible aceptar la hipótesis de que existe linealidad de $\mathrm{E}(\mathrm{Y} \mid \mathrm{P}(\mathrm{Z})=\mathrm{p}, \mathrm{X}=\mathrm{x})$. Recursivamente, se llega hasta una prueba con la quinta potencia, donde el parámetro de P5 no es significativo (al 95\% de confianza).

En la medida que se aumenta la potencia del polinomio, aumenta la posibilidad de que exista colinealidad dentro de la expresión polinómica. Sin embargo, la prueba permite validar la presencia de heterogeneidad antes de que se produzcan problemas de especificación en la regresión. En consecuencia, la ecuación de ingresos puede ser especificada con un polinomio en $\mathrm{p}$ hasta la cuarta potencia.

$\mathrm{Al}$ afirmar que no es posible rechazar la hipótesis formulada sobre una forma polinomial del MTE, se obtiene la evidencia deseada. Por lo tanto, puede concluirse que se está ante la presencia de heterogeneidad esencial, y a continuación, se analizan las implicancias de este resultado. 
Cuadro 2: Test por presencia de Heterogeneidad Esencial Método - Linealidad del valor esperado condicional $\mathrm{E}(\mathrm{Y} \mid \mathrm{P}(\mathrm{Z}), \mathrm{X})$. Fuente: Elaboración en base a Encuesta de Hogar CASEN 2015, MDS (2016) $^{7}$

\begin{tabular}{crrrrr}
\hline Variable & Lineal & Cuadrático & \multicolumn{1}{c}{ Cúbico } & \multicolumn{1}{c}{$\begin{array}{c}\text { Cuarta } \\
\text { potencia }\end{array}$} & $\begin{array}{c}\text { Quinta } \\
\text { potencia }\end{array}$ \\
\hline P & 2,4196 & 2,8923 & 4.9751 & 7.0951 & 7.4662 \\
& $(0,1649)$ & $(0,1980)$ & $(0,2800)$ & $(0,3287)$ & $(0.3930)$ \\
P2 & & $-0,85728$ & $-5,9473$ & -21.0584 & -25.1970 \\
& & $(0,1987)$ & $(0,5236)$ & $(1,3398)$ & $(2.7510)$ \\
P3 & & 3,9085 & 31.1376 & 44.6444 \\
& & & $(0,3720)$ & $(2.2537)$ & $(8.1592)$ \\
P4 & & & -15.8049 & -33.2526 \\
& & & & $(1.2902)$ & $(10.2116)$ \\
P5 & & & & $7.8331^{8}$ \\
& & & & & $(4.5477)$ \\
F9 & 1093,85 & 1055,89 & 1025,92 & 1000,22 & 968.09 \\
& $(0,0000)$ & $(0,0000)$ & $(0,0000)$ & $(0,0000)$ & $(0,0000)$ \\
\hline
\end{tabular}

\section{Ingresos y el estimador MTE ante la presencia de heterogeneidad esencial}

En base a la prueba de linealidad del estimador, se obtiene como resultado una forma polinómica (Ecuación 12) que relaciona los ingresos percibidos, las características determinantes observadas de una persona $(X)$ y su probabilidad de trabajar cumpliendo requisitos de formalidad, medido a través del puntaje de propensión.

$$
\mathrm{E}(\ln W \mid \mathrm{X}=\mathrm{x}, \mathrm{P}(Z)=\mathrm{p})=\alpha_{0}+\beta_{0} X+\left(\left(\beta_{1-} \beta_{0}\right) X\right) p+\phi_{1} p+\phi_{2} p^{2}+\phi_{3} p^{3}+
$$

\footnotetext{
7 Errores estándar entre paréntesis.

${ }^{8} \mathrm{P}>|\mathrm{t}|=0,08>0,05 \mathrm{p}$-valor $<0,05$

${ }_{9}^{9}$ Hipótesis conjunta de los coeficientes de los polinomios de mayor orden del puntaje de propensión p.
} 
La predicción obtenida por este modelo, al contener un componente polinómico de potencia cuatro en $\mathrm{p}$, arroja como resultado que los Ingresos esperados ( $\ln W$ en escala logarítmica) muestra la variación descrita en el gráfico 5 , conforme varía la probabilidad de estar trabajando de manera formal $\mathrm{P}(\mathrm{Z})$.

En el gráfico 5 se observa que los ingresos estimados por este modelo polinómico registran sus mayores valores en dos rangos de probabilidades. El primero, como es esperable, se encuentra cercano a la probabilidad de ser trabajador formal (a la derecha del eje de la ordenada), pero también lo es en un segundo rango, ubicado entre el primer y cuarto decil de $\mathrm{P}(\mathrm{Z})$, sector en el cual el individuo tiene una mayor probabilidad de estar trabajando de manera informal.

Gráfico 5: Estimación del Logaritmo de los Ingresos a partir de expresión polinómica del Puntaje de Propensión aplicado sobre Trabajadores Formales e Informales 2015. Fuente: Elaboración en base a Encuesta de Hogar CASEN 2015, MDS (2016)

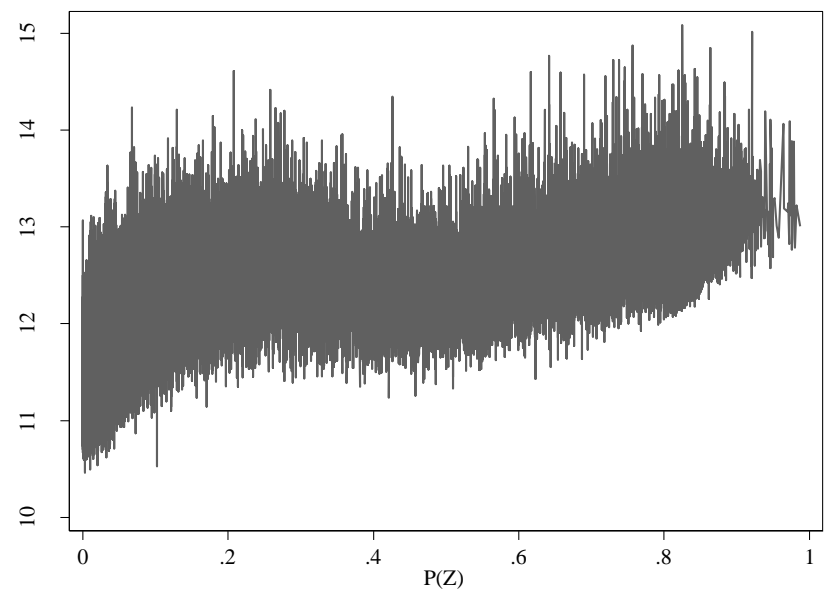

Teniendo en consideración que la ecuación de ingresos puede ser expresada en términos de $\mathrm{P}(\mathrm{Z})=p$ bajo la forma polinómica de potencia cuatro, y que el estimador MTE corresponde a la primera derivada en $p$ del modelo, la forma que adopta entonces es la de un polinomio de grado tres, cuyos coeficientes pueden ser estimados.

$$
\operatorname{MTE}\left(x, U_{D}\right)=\frac{\partial\left(\ln W_{i} \mid X_{i}=x_{i}, P(Z)=p\right)}{\partial p}
$$




$$
=\left(\left(\beta_{1-} \beta_{0}\right) X\right)+\phi_{1}+2 \phi_{2} p+3 \phi_{3} p^{2}+4 \phi_{4} p^{3}
$$

Si se aísla en la ecuación del estimador MTE los componentes observados de los no observados (MTEU), se obtiene una relación sin la variabilidad presente en las observaciones individuales, lo que facilita el análisis (véase el gráfico 6).

Gráfico 6: Estimador MTE y MTEU (X, P) calculado sobre muestra de Trabajadores Formales e Informales. 2015. Fuente: Elaboración en base a Encuesta de Hogar CASEN 2015, MDS (2016)
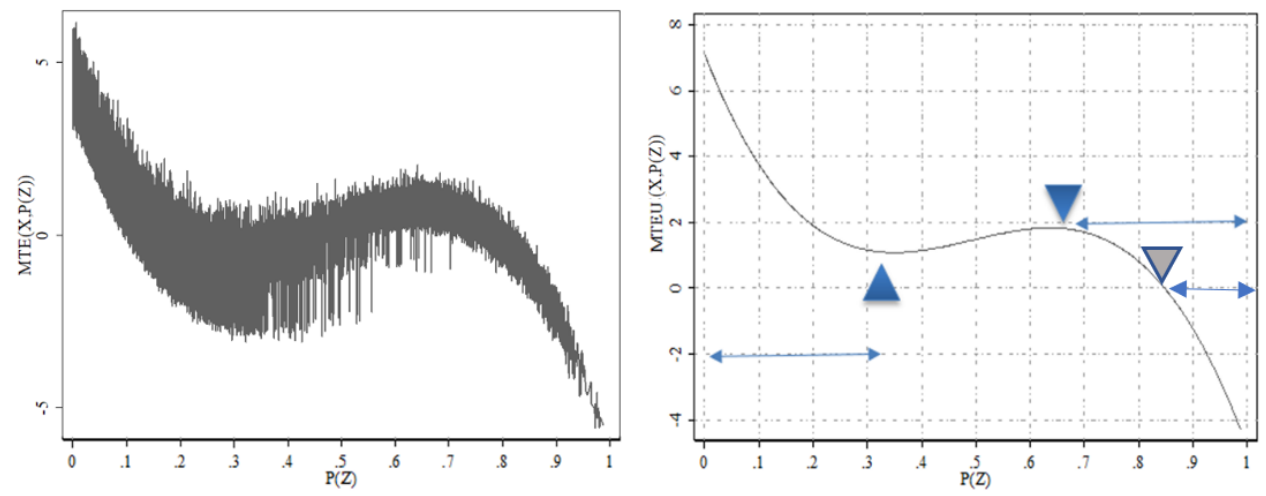

Como resultado, se obtiene un estimador MTE que no es ortogonal en UD o $\mathrm{P}(\mathrm{Z})$. Su no linealidad, permite inferir que los cambios marginales tampoco son constantes. La forma polinómica resultante, provee información respecto de cómo se comportan las variaciones infinitesimales en los ingresos debido a un cambio desde un trabajo informal a uno formal.

Estas variaciones, conforme se modifica la variable $\mathrm{P}(\mathrm{Z})$, se obtienen dentro de un rango que va desde [0], probabilidad de trabajar de manera informal, hasta [1] probabilidad de trabajar cumpliendo requisitos de formalidad.

La relación entre la forma del MTEU y el P(Z) es relevante, pues según Natalia Radchenko $(2014,2016)$ valores elevados y bajos muestran las mínimas ganancias o pérdidas por características no observables que motivan al individuo trabajar de manera formal por sobre una alternativa de trabajo informal o viceversa, lo que constituye una 
evidencia de la voluntad marginal a pagar, o las ganancias (pérdidas) marginales por parte del individuo para trabajar bajo alguna de estas modalidades.

Si se analiza el comportamiento de la curva, en un extremo del intervalo cercano al origen, se encuentran los casos de altas probabilidades de trabajar de manera informal, coinciden con valores más elevados del MTE, es decir, la brecha de ingresos es mayor. En este tramo el MTE tiene un valor positivo, pero decrece. La variación negativa implica que existe ordenamiento por ganancias y los agentes tienen en consideración ventajas comparativas de una localización en diferentes modalidades de empleo (Radchenko, 2014).

Esto puede ser interpretado como la selección por parte de los agentes, quienes se encuentran eligiendo trabajar de manera idiosincrática, poniendo en evidencia la existencia de un componente de elección voluntaria del sector elegido, lo que es posible cuando el mercado del trabajo no impone o tiene bajas barreras para asumir una actividad laboral en cualquiera de los dos segmentos.

Asimismo, dentro del intervalo, existen dos puntos de inflexión, en el primero, se marca el cambio desde una forma cóncava decreciente a un convexo creciente; y en el segundo, la curva vuelve a cambiar a una forma cóncava decreciente, tramo donde cruza el eje de la abscisa hacia valores negativos.

Entre ambos puntos de inflexión, se observa un tramo constituido por agentes que tienen, dadas sus características, probabilidades de modalidad de trabajo (formal-informal). En este rango, la trayectoria decreciente se revierte, y se produce un aumento del MTEU conforme $\mathrm{P}(\mathrm{Z})$ aumenta.

Esto puede interpretarse, según lo que señala Radchenko (2014) como la presencia de una correlación negativa entre las ganancias basadas en características no observables y la ecuación de selección. De hecho, en el Gráfico $\mathrm{N}^{\circ} 5$ de ingresos estimados, se observa que ellos alcanzan, en promedio, un menor nivel respecto de quienes se ubican hacia los extremos de la gráfica, que corresponden a los casos de mayor probabilidad de trabajo informal o formal respectivamente.

Al otro extremo del intervalo, cercano a la probabilidad de trabajar formalmente, cuando $\mathrm{P}(\mathrm{Z})$ o UD superan un valor de 0,65 , el MTEU alcanza un nuevo punto de inflexión, adoptando una trayectoria cóncava y decreciente, a partir del valor 0.85 alcanza el eje de la abscisa, adoptando a partir de este punto valores negativos ${ }^{10}$. Esta gráfica

\footnotetext{
${ }^{10}$ Basado en características no observables, manteniendo fijas las variables observadas.
} 
representa la primera derivada desde la gráfica de ingresos, su evolución corresponde a su pendiente, su forma decreciente refleja el crecimiento a tasa marginal decreciente de los ingresos estimados (lnW, logaritmo del ingreso) y muestra una disminución de la brecha de ingresos, que puede estar reflejando el efecto del pago de impuestos y seguridad social. (Radchenko, 2016).

Ante la presencia de heterogeneidad esencial, y a modo de conclusión se puede inferir que existe selección y voluntariedad en la elección de la modalidad de trabajo. Puesto que las personas están respondiendo al tratamiento seleccionando la condición de trabajo basados en ganancias o recompensas idiosincráticas. Lo que implica que se encuentran revelando una variación conductual que las lleva a elegir la modalidad de trabajo.

\section{Comparación de resultados con el uso de estimadores de efectos calculados mediante métodos alternativos}

Luego de obtener los resultados de una estimación de los ingresos de trabajadores formales usando el Efecto Marginal del Tratamiento (MTE), otros medios de estimación del efecto del tratamiento utilizados comúnmente son:

- $\quad$ El Efecto Promedio del Tratamiento (Average Treatment Effect). $\mathrm{ATE}=\mathrm{E}\left(\ln W_{1}-\ln W_{0}\right)$.

- El Tratamiento promedio sobre los Tratados (ATET, Average Treatment Effect over Treated). ATET $=\mathrm{E}\left(\ln W_{1}-\ln W_{0} \quad \mathrm{D}=\right.$ 1).

- El Tratamiento sobre los No Tratados (ATUT, Average Treatment Over Untreated $)$ ATUT $=\mathrm{E}\left(\ln W_{1}-\ln W_{0} \mid \mathrm{D}=0\right)$.

Todos ellos corresponden a combinaciones lineales del MTE (Heckman, Urzúa y Vytlacil, 2006), y son utilizados como complemento de la estimación realizada dentro de la etapa precedente. Para ello, se usan cuatro métodos de cálculo alternativos: el ajuste de regresión, el ajuste de regresión con media condicional exponencial, la probabilidad inversa ponderada y el emparejamiento por vecino más cercano.

El ajuste de regresión (RA, Regression Adjustment) corresponde a un modelo de selección, los estimadores de RA ejecutan regresiones separadas para cada nivel de tratamiento, las medias de los resultados 
predichos utilizando todos los datos, se estima los coeficientes de las medias de los potenciales resultados (POM, Potential Outcome Means), evaluados para cada nivel de tratamiento, y luego se utilizan las diferencias de los resultados obtenidos (condicionales en los POM).

La Probabilidad Inversa Ponderada (IPW, Inverse Probability Weighted) corresponde a un modelo de tratamiento, en donde se pesan las observaciones sobre la variable de resultados, utilizando el inverso de la probabilidad que sea observada, considerando el proceso de falta de datos producto del agrupamiento no aleatorio de las muestras, clasificadas en función del tratamiento.

El método de emparejamiento por el vecino más cercano (Nearest Neighbor Matching, NNM), basa sus estimadores en el puntaje de propensión, utilizando un promedio de los resultados de las personas más cercanas para imputar el resultado potencial faltante para cada individuo muestreado. Este método corresponde a una técnica no paramétrica, y no se asume a priori ninguna forma funcional para el parámetro del modelo de resultados. La diferencia entre el resultado observado y el resultado potencial imputado corresponde a una estimación del tratamiento esperado a nivel individual, condicional sobre las covariables observadas.

Los estimadores son calculados mediante apoyo computacional ${ }^{11}$. Los resultados se presentan en el cuadro 3.

En la segunda columna, el valor de 0,71 (en escala logarítmica) corresponde a la diferencia de las medias de los ingresos obtenidas mediante mínimos cuadrados ordinarios a las submuestras de personas con empleo formal e informal.

Luego, se obtienen los estimadores ATE y ATET usando diferentes métodos de cálculos, como resultado se observa una notoria reducción de la brecha de ingresos entre ambas categorías de empleo, a un rango de 0,23 a 0,25 (en escala logarítmica, €106,7). Este resultado es coincidente con lo señalado por Heckman y Vytlacil, (2005), quienes afirman que el uso de MCO introduce un sesgo al alza en los resultados. Incluso, Cunningham y Maloney (2001) y Maloney (2002), relativizan el uso de comparaciones de ingresos como medida adecuada, debido a la existencia de factores no observados que pueden incidir en los resultados. Según estos autores, esto puede deberse a emprendimientos en etapa de maduración o el efecto de otros beneficios no pecuniarios.

11 Cálculos son realizados mediante el uso de software Stata. 
Cuadro 3: Estimación de efectos de tratamiento promedio ATE y ATET. Fuente: elaboración en base a Encuesta de Hogar CASEN 2015, MDS. ${ }^{2}$

\begin{tabular}{|c|c|c|c|c|c|}
\hline & \multicolumn{5}{|c|}{ Valores estimados, escala logarítmica. } \\
\hline $\begin{array}{l}\text { Efecto de } \\
\text { tratamiento. }\end{array}$ & $\begin{array}{l}\text { Diferencia } \\
\text { de Medias } \\
\text { Formal/ } \\
\text { Informal }\end{array}$ & $\begin{array}{l}\text { Ajuste de } \\
\text { Regresión } \\
(\mathrm{AR})\end{array}$ & $\begin{array}{l}\text { AR con la } \\
\text { media } \\
\text { condicional } \\
\text { exponencial }\end{array}$ & $\begin{array}{l}\text { Probabilidad } \\
\text { Inversa } \\
\text { Ponderada } \\
\text { (IPW) }^{13}\end{array}$ & $\begin{array}{l}\text { Emparejamiento } \\
\text { por Vecino más } \\
\text { Cercano }\end{array}$ \\
\hline ATE & $\begin{array}{l}0,7125 \\
(0,00521)\end{array}$ & $\begin{array}{l}0,2419 \\
(0,00009)\end{array}$ & $\begin{array}{l}0,2411 \\
(0,00094)\end{array}$ & $\begin{array}{l}0,2580 \\
(0,02189)\end{array}$ & $\begin{array}{l}0,2336 \\
(0.00031)\end{array}$ \\
\hline ATET & --- & $\begin{array}{l}0,2215 \\
(0,00112)\end{array}$ & $\begin{array}{l}0,2188 \\
(0, .001143)\end{array}$ & $\begin{array}{l}0,1488 \\
(0.01207)\end{array}$ & $\begin{array}{l}0,2256 \\
(0,00033) \\
* *\end{array}$ \\
\hline
\end{tabular}

Los diferentes estimadores coinciden en que la brecha entre ingresos formales e informales es mayor para la muestra que incluye al conjunto de trabajadores (ATE) respecto del obtenido cuando la brecha es observada en los trabajadores formales (ATET). En consecuencia, si ATE $>$ ATET, el aumento que refleja el efecto promedio del total de la muestra ATE se debe a la presencia de los casos provenientes de la submuestra de los trabadores informales, por lo tanto, se podría inferir que el estimador ATUT tiene un valor mayor, aunque no ha sido calculado directamente.

Suponiendo que un ordenamiento ATUT $>$ ATE $>$ ATET se debería al efecto de características no observadas, puede interpretarse que existiría una mayor ventaja pecuniaria para quienes ejercen un trabajo informal al formalizarse, que la que obtendrían los trabajadores formales por dejar de serlo.

Junto con la obtención de los estimadores de efectos ATE y ATET, también puede estimarse la media de ingresos promedios estimados. En esta oportunidad, se ha seleccionado el estatus Sin Tratamiento, el cual corresponde a la media de los ingresos que se obtienen trabajando de manera informal.

\footnotetext{
12 Valores entre paréntesis corresponden al margen de error estándar.

13 Soporte Común corresponde a 42.348 casos, 38,3 por ciento de la muestra original.
} 
Cuadro 4: Estimación de ingresos medios de categoría sin tratamiento usando estimador ATE y ATET. Fuente: Elaboración en base a Encuesta de Hogar CASEN 2015, MDS (2016) ${ }^{14}$

\begin{tabular}{lllll}
\hline \multirow{2}{*}{$\begin{array}{l}\text { Estimación de } \\
\text { media de ingresos }\end{array}$} & \multicolumn{4}{c}{ Valores estimados, escala logarítmica } \\
\cline { 2 - 5 } $\begin{array}{l}\text { sin tratamiento } \\
\text { (Trabajo informal) }\end{array}$ & $\begin{array}{l}\text { Diferencia } \\
\text { de Medias }\end{array}$ & $\begin{array}{l}\text { Ajuste de } \\
\text { Regresión }\end{array}$ & $\begin{array}{l}\text { AR con la } \\
\text { media }\end{array}$ & $\begin{array}{l}\text { Probabilidad } \\
\text { Fonversa }\end{array}$ \\
& Informal & & $\begin{array}{l}\text { condicional } \\
\text { exponencial }\end{array}$ & $\begin{array}{l}\text { Ponderada } \\
(\text { IPW })^{15}\end{array}$ \\
Usando estimador & 12,1051 & 12,5192 & 12,5212 & 12.2496 \\
ATE 16 & $(0,0044)$ & $(0,0009)$ & $(0,0009)$ & $(0,0060)$ \\
Usando Estimador & $\cdots$ & 12,6436 & 12,64635 & 12,5444 \\
ATET & & $(0,0011)$ & $(0,001141)$ & $(0,01208)$ \\
\hline
\end{tabular}

Como resultado, las estimaciones de ingresos medios expresados en escala logarítmica fluctúan según el método utilizado entre un rango de 12.1 a 12.6, expresados en pesos chilenos, equivalen a un rango de CLP $\$ 179.871$ a $\$ 296.558$ (€254 a €419) ${ }^{17}$.

A modo de referencia, el salario mínimo legal existente en Chile en julio de 2015 era de CLP $\$ 241.000$ (€304,6), una persona que recibe como remuneración esta cantidad, una vez descontados los aportes previsionales reduce el ingreso líquido de $\$ 170.340$ (€240,7), por lo tanto, los resultados de las estimaciones dejan en evidencia que las y los trabajadores informales estarían generando ingresos superiores al ingreso líquido que reciben quienes perciben el salario mínimo.

\section{2. ¿Es posible generalizar este resultado?}

Para responder a esta pregunta, se aplica la prueba de linealidad del estimador a una submuestra compuesta por las personas provenientes del primer quintil socioeconómico (20 por ciento más pobres). Los datos que cumplen el soporte común reducen la muestra a 7.635 casos, cerca de un 7 por ciento del total. En este segmento, según la clasificación adoptada, la proporción de trabajadores informales se

${ }^{14}$ Valores entre paréntesis corresponde al margen de error.

${ }_{15} \mathrm{Al}$ igual que en cuadro 3 , el soporte común corresponde a 42.348 casos, 38,3 por ciento de la muestra original.

${ }^{16}$ En el cálculo del ATE, los POM (Potential Outcome Means, o Media de Resultados Potenciales son evaluados en dos estados: 0 informal y 1 formal), por defecto están generados para la base de sujetos no tratados.

17 Tipo de cambio 1 euro = 707,6 CLP pesos chilenos, 30 junio 2015 . 
eleva a un 49,6 por ciento del total, cifra que duplica a la proporción del total de la población y triplica de la cantidad de trabajadores informales que registra el quinto quintil.

Se aplica el mismo procedimiento iterativo realizado para la muestra completa, sólo que esta vez se restringe a casos del primer quintil, como resultado, al agregar una expresión cuadrática el coeficiente resulta no significativo (pvalor=0,434). Sólo al agregar el termino cúbico, los coeficientes de expresiones cuadrática y cúbica son significativas. Sin embargo, algunas variables, tales como experiencia, tamaño de empresa y el coeficiente del término $\left(\left(\beta_{1-} \beta_{0}\right) X\right) p$ dejan de ser significativas. En consecuencia, los resultados deben interpretarse con cautela, producto de que la evidencia es débil y no es posible afirmar que existe heterogeneidad esencial en este segmento.

Este resultado permitiría inferir que se está en presencia de un estimador lineal, en donde, cada agente está ejerciendo una actividad laboral consistente con el puntaje de propensión que se le asigna en función de las características conocidas dentro del proceso de asignación. $\mathrm{Al}$ no haber muestras de una elección distinta, podría interpretarse el resultado como señal de la presencia de barreras para acceder a trabajos formales, las cuales estarían inhibiendo respuestas idiosincráticas. Una condición más cercana a un mercado dual, en el sentido planteado por Harris y Todaro (1970), el cual se encontraría constituido por un segmento de trabajadores formales, y otro que genera sus ingresos mediante actividades económicas informales.

\section{Conclusiones}

En primer lugar, para el desarrollo de esta investigación se ha formulado y aplicado un método particular de medición de la informalidad laboral, que se basa en recomendaciones formuladas por la OIT, y que ha sido adaptado al marco regulatorio chileno. Se ha aplicado esta fórmula a los datos de las encuestas de hogares CASEN (2009 a 2016). Con los datos recopilados en el año 2015 y publicados en 2016, se realiza una caracterización del trabajo informal y se formula la hipótesis de la investigación, por lo tanto, debe precisarse que los principales resultados obtenidos son válidos para este período de tiempo.

La aplicación de esta nueva metodología de clasificación permite estimar la informalidad laboral, la cual alcanzó el año 2015 un 26,2 por ciento de la población ocupada (1.974.770) personas, cifra compuesta por 
cerca de un sesenta por ciento de trabajadores autónomos y un cuarenta por ciento de trabajadores asalariados.

En el caso de los trabajadores autónomos, cerca de 27,8 por ciento registra un cumplimiento total o parcial de la normativa tributaria que rige sus actividades. Por el contrario, dentro de los asalariados, un 13,8 por ciento lo hacen sin contar con un contrato de trabajo escrito, motivo por el cual, las políticas públicas destinadas a una disminución del trabajo informal deberían incluir las acciones que permitan asegurar un trabajo asalariado bajo condiciones de formalidad de manera prioritaria.

El foco de la investigación ha sido poder determinar si el trabajo informal responde a una condición obligada o a una opción voluntaria, esto se indaga mediante la aplicación una metodología de selección y tratamiento, formulada por Heckman, Urzúa y Vytlacil (2006), utilizando métodos de puntaje de propensión para estudiar los efectos del tratamiento heterogéneo en individuos con diferentes propensiones a ser tratados.

La aportación realizada por Radchenko $(2014,2016)$ ha permitido adaptar el concepto de tratamiento al de la elección del tipo de trabajo, permitiendo así, su aplicación en el objetivo de la investigación.

Como principal resultado, se detecta la presencia de heterogeneidad esencial en la obtención de ingresos. Esto implicaría que la distribución de los efectos de tratamiento en los individuos, definido éste como el adoptar una modalidad de trabajo formal, al ser relacionado con el proceso de asignación, revelaría una variación conductual que está llevando a una persona a trabajar en un segmento distinto al que debería, según sus características observadas.

Esto permitiría inferir que los agentes presentan una respuesta idiosincrática sobre la elección de la modalidad de trabajo que adoptan. La cual puede basarse en relaciones del tipo beneficio - costo o a características no observadas. La posibilidad de que estén haciendo efectiva la elección, implicaría que el mercado laboral presenta bajas barreras para acceder a la formalidad, o para ejercer una actividad informal, configurando una estructura integrada y competitiva.

Cuando se utiliza un estimador insesgado, se obtiene como resultado que la media de los ingresos del trabajo informal es superior al salario mínimo legal, lo que evidencia que un grupo de quienes ejercen este tipo de actividades obtendrían ventajas monetarias del incumplimiento total o parcial de la regulación.

Sin embargo, no es posible generalizar una conclusión sobre la presencia de heterogeneidad esencial, puesto que, al aplicar una prueba similar a los datos de la muestra que provienen del quintil de personas 
de menores ingresos, la evidencia es menos concluyente, pero apunta hacia la ortogonalidad entre el estimador y el intervalo de probabilidades. Situación que se asocia con la presencia de barreras que impedirían la elección del tipo de empleo para este segmento.

La presencia simultánea de ambos resultados constituye un tipo de heterogeneidad, con la presencia simultánea de agentes que eligen de manera voluntaria un trabajo formal o informal, junto con quienes se ven sometidos a barreras para acceder a la formalidad y terminan ejerciendo una actividad informal de manera forzada.

\section{Bibliografía}

Arcand, J. y Bassole, L. (2011). "Essential Heterogeneity in the Impact of Community Driven Development" (WIP).

Castells, M., Portes, A. y Benton, L. (1989). "The informal economy: studies in advanced countries and less developed economies". Londres. The Johns Hopkins University Press.

De Soto, H. (1989). "The other path. The invisible revolution in the third world". New York: Harpers \& Row Publishers.

Fields, G. (2005). "A guide to multisector labour market models". Social Protection Discussion Paper Series. No. 0505, April 2005. Social Protection Unit, Human Development Network, the World Bank.

Günther, I. y Launov, A. (2006). "Competitive and segmented informal labour markets". The Institute for the Study of Labor (IZA) in Bonn, Discussion Paper No. 2349, September 2006.

Hart, K. (1973). "Informal Income Opportunities and Urban Employment in Ghana”. The Journal of Modern African Studies / Volume 11 / Issue 01 / March 1973, pp 61 - 89.

Heckman, J. y Vytlacil, E. (2005). "Structural equations, treatment effects, and Econometric policy evaluation". Econometrica, Vol. 73, No. 3 (mayo, 2005), 669-738. 
Heckman, J., Schmierer, D. y Urzua, S. (2007). "Testing for Essential Heterogeneity" (Draft).

Heckman, J., Urzua, J. y Vytlacil, E. (2006). "Estimation of Treatment Effects under Essential Heterogeneity". University of Chicago.

Instituto de Previsión Social y Facultad Latinoamericana de Ciencias Sociales (2017), "Determinación de la incidencia del trabajo informal en el ámbito previsional y de seguridad social, con enfoque de género". Pág. 108.

León, V. (2017) "Heterogeneidad en el mercado de trabajo informal en Chile", Universidad de Alcalá.

Maloney, W. (2002). "Informality Revisited". Policy Research Working Paper 2965 The World Bank.

Mincer, J. (1958). "Investment in Human Capital and Personal Income Distribution”. Journal of Political Economy 66 (4): 281-302

Ministerio de Desarrollo Social. (2016). "Encuesta de Caracterización Socioeconómica Nacional (CASEN) 1990”, 2015. Santiago.

Organización Internacional del Trabajo. (1972). "Employment, incomes and equality. Strategy for increasing productive employment in Kenya".

Organización Internacional del Trabajo. (1993). "Resolución sobre las estadisticas del empleo en el sector informal, adoptada por la decimoquinta Conferencia Internacional de Estadisticos del Trabajo. Conferencia Internacional de Estadísticos del Trabajo".

Organización Internacional del Trabajo. (2002). "El trabajo decente y la economía informal, Informe VI, Conferencia Internacional del Trabajo $90^{a}$ Reunión 2002'.

Organización Internacional del Trabajo. (2003). "Decimoséptima Conferencia Internacional de Estadísticos del Trabajo”.

Organización Internacional del Trabajo. (2012). "Statistical update on employment in the informal economy" Oficina de Estadísticas. 
Pagés-Serra C. y Madrigal, L. (2008). "Is informality a good measure of job quality? Evidence from job satisfaction data". Washington: Banco Interamericano de Desarrollo. Working Paper nº 654 Diciembre 2008.

Perry, G., Maloney, W., Arias, O., Fajnzylber, P., Mason, A. y Saavedra, J. (2007). "La informalidad: escape y exclusión". Washington, World Bank.

Portes, A. y Heller, W. (2004). "La economía informal". Santiago de Chile: CEPAL, Serie Políticas Sociales N. ${ }^{\circ}$ 100. Santiago.

PREALC. (1978). "Sector Informal, funcionamiento y políticas". (Tokman, V. Editor) Oficina Internacional del Trabajo.

Radchenko, N. (2014). "Heterogeneity in Informal Salaried Employment: Evidence from the Egyptian Labor Market Survey". World Development Vol 62, 169-188.

Radchenko, N. (2016). "Informal Employment in Developing Economies: Multiple Heterogeneity”. The Journal of Development Studies.

Rosenbaum P. y Rubin D. (1983) "The Central Role of the Propensity Score in Observational Studies for Causal Effects”. Biometrika.

Tokman, V. (2004). "Una voz en el camino. empleo y equidad en América Latina: 40 años de búsqueda". Santiago: Fondo de Cultura Económica.

Villagómez, V. (2002). "Oferta de trabajo familiar y segmentación del mercado laboral: El caso de México". Secretaría del Trabajo y Previsión Social, Gobierno de México.

Wooldridge, J. (2009). "Introductory Econometrics: A Modern Approach". 5th Edition.

Zhou, X., y Yu, X. (Feb de 2016). "Propensity Score-Based Methods versus MTE-Based Methods in Causal Inference: Identification, Estimation, and Application". Sociological Methods Research. 